\title{
All-in-one Lewis Base for Enhanced Precursor and Device Stability in Highly Efficient Perovskite Solar Cells
}

Jun Zhu, ${ }^{1, \$}$ Dong Hoe Kim, ${ }^{2,3 \$}$ Ji Dong Kim, ${ }^{2}$ Dong Geon Lee, ${ }^{4}$ Won Bin Kim, ${ }^{4}$ Shi wang Chen, ${ }^{4}$ Jun Young Kim, ${ }^{4}$ Jae Myeong Lee, ${ }^{4}$ Hyemin Lee, ${ }^{2}$ Gill Sang Han,,${ }^{4}{ }^{*}$ Tae Kyu Ahn ${ }^{1, *}$ and Hyun Suk Jung ${ }^{4, *}$

${ }^{1}$ Department of Energy Science, Sungkyunkwan University (SKKU), Suwon, 16419, Republic of Korea

${ }^{2}$ Department of Nanotechnology and Advanced Materials Engineering, Sejong University, Seoul 05006, Republic of Korea

${ }^{3}$ Department of Materials Science and Engineering, Korea University, Seoul 02841, Republic of Korea

${ }^{4}$ School of Advanced Materials Science \& Engineering, Sungkyunkwan University (SKKU), Suwon 16419, Republic of Korea

\section{Materials}

Fluorine-doped tin oxide $\left(\mathrm{SnO}_{2}: \mathrm{F}, \mathrm{FTO}\right)$ was purchased from Pilkington (TEC8, $\left.8 \Omega \mathrm{sq}^{-1}\right)$. Titanium tetrachloride $\left(\mathrm{TiCl}_{4}\right)$, dimethyl sulfoxide (DMSO, 99.9\%), acetonitrile $(99.8 \%)$, dimethylformamide (DMF, 99.8\%), chlorobenzene (CB, 99\%), diethyl ether (99.7\%), 4,4'(hexafluoroisopropylidene)diphthalic anhydride (6-FDA, Sigma-Aldrich, 99\%), 4,4carbonyldiphthalic anhydride (CDA, Sigma-Aldrich, 98\%), $\mathrm{PbI}_{2}$ (99.995\%), $\mathrm{PbBr}_{2}$ (99\%), $\mathrm{HC}\left(\mathrm{NH}_{2}\right)_{2} \mathrm{I}$ (FAI), $\mathrm{CH}_{3} \mathrm{NH}_{3} \mathrm{Br}$ (MABr), methylammonium chloride (MACl), lithium bis(trifluoromethanesulfonyl)imide salt (Li-TFSI), and 4-tert-butylpyridine (tBP) were purchased from Sigma-Aldrich (USA). 2,2',7,7'-tetrakis(N,N-di-p-methoxyphenylamine)-9,9'spirobifluorene (Spiro-OMeTAD, LT-S922) was purchased from Luminescence Technology Corp.. All materials were purchased from commercial sources and used as received without further purification. 


\section{Device Fabrication}

FTO-coated glass substrates were etched via laser irradiation and then cleaned via sonication with deionized water, ethanol, and acetone for $15 \mathrm{~min}$ each. The $2 \mathrm{M}$ aqueous $\mathrm{TiCl}_{4}$ stock solution was first prepared by mixing $\mathrm{TiCl}_{4}$ with deionized water in an ice bath, and then stored in a refrigerator at $-4{ }^{\circ} \mathrm{C}$, which remained stable for at least three months. $\mathrm{A} \mathrm{TiO}_{2}$ compact layer was then deposited on the substrates by chemical bath deposition. The cleaned FTO substrate was briefly soaked in $0.18 \mathrm{M}$ aqueous $\mathrm{TiCl}_{4}$ solution and placed in a sealed glass container. The glass container was then placed into a drying oven at $70{ }^{\circ} \mathrm{C}$ for $45 \mathrm{~min}$. After cooling, the FTO substrate was repeatedly rinsed using ethanol and deionized water three times to remove any residual on the surface, then annealed at $200{ }^{\circ} \mathrm{C}$ for $1 \mathrm{~h}$, and cooled to room temperature under ambient conditions.

The ratio of the Lewis base additive is related to the perovskite precursor solution concentration $(1.6 \mathrm{M})$ and is denoted as $\mathrm{x} \%$ FDA and $\mathrm{y} \% \mathrm{CDA}$, where $\mathrm{x}$ and $\mathrm{y}$ represent the molar contents of additives. The control device had no additives. The $1.6 \mathrm{M}$ $\left(\mathrm{FAPbI}_{3}\right)_{0.95}\left(\mathrm{MAPbBr}_{3}\right)_{0.05}$ perovskite precursor solutions were prepared by dissolving corresponding amounts of FAI (0.263 g), MABr (0.009 g), $\mathrm{PbI}_{2}(0.726 \mathrm{~g}), \mathrm{PbBr}_{2}$ (0.029 g), and $\mathrm{MACl}(0.035 \mathrm{~g})$ in $1 \mathrm{~mL}$ of a DMSO/DMF (8/1) mixed solvent (with $3 \%$ molar ratio $\mathrm{PbI}_{2}$ excess). When the additive was added to $1.6 \mathrm{M}$ perovskite precursor solutions, the molar ratios of FDA and CDA increased from $0.00 \%$ (control), to $0.025 \%, 0.050 \%, 0.10 \%$, $0.20 \%$, and $0.30 \%$, respectively. The compact $\mathrm{TiO}_{2} / \mathrm{FTO}$ substrate was coated with the perovskite precursor solution using a one-step antisolvent spin-coating method. First, the substrates were exposed to UV-ozone irradiation for $30 \mathrm{~min}$, and the dissolved solution was spin-coated on the substrates at 5,000 rpm for $25 \mathrm{~s}$ and $0.55 \mathrm{~mL}$ ether was quickly dripped on the rotating substrate $20 \mathrm{~s}$ after the spin-procedure started. The semitransparent film was heated at $150{ }^{\circ} \mathrm{C}$ for $10 \mathrm{~min}$ to obtain a dense perovskite film. After cooling to room temperature under ambient conditions, $30 \mu \mathrm{L}$ of HTM was deposited by spin coating at 4,000 rpm for $30 \mathrm{~s}$. The HTM solution contained $90 \mathrm{mg}$ of spiro-OMeTAD, $36 \mu \mathrm{L}$ of 4-tert-butylpyridine, and $23 \mu \mathrm{L}$ of $520 \mathrm{mg} \mathrm{mL}^{-1}$ lithium bis(trifluoromethylsulphonyl)imide acetonitrile solution dissolved in $1 \mathrm{~mL}$ of $\mathrm{CB}$. For the thermal stability test, PTAA was used instead of spiro-OMeTAD, which was prepared by dissolving $20 \mathrm{mg}$ of PTAA in toluene mixed with $4.0 \mu \mathrm{L}$ of tBP and $3.20 \mu \mathrm{L}$ of 1.8 M Li-TFSI solution (dissolved in acetonitrile). An amount of $30 \mu \mathrm{L}$ of PTAA stock solution was spin-coated on the as-prepared perovskite film at 4,000 rpm for $25 \mathrm{~s}$. Finally, an Au electrode metal with $50 \mathrm{~nm}$ thickness was thermally evaporated on top of the device to form the back contact at $0.4 \AA \mathrm{s}^{-1}$. 


\section{Synthesis of adduct powder:}

To synthesize the $\left(\mathrm{FAPbI}_{3}\right)_{0.95}\left(\mathrm{MAPbBr}_{3}\right)_{0.05} \cdot \mathrm{DMSO}$ adduct powder, the corresponding amounts of FAI (0.263 g), $\mathrm{MABr}(0.009 \mathrm{~g}), \mathrm{PbI}_{2}(0.726 \mathrm{~g})$, and $\operatorname{PbBr}_{2}(0.029 \mathrm{~g})$ were dissolved in $1 \mathrm{~mL}$ of DMSO/DMF (8/1) mixed solvent and heated at $70{ }^{\circ} \mathrm{C}$ for $30 \mathrm{~min}$. The solution was added dropwise to $15 \mathrm{~mL}$ of diethyl ether, and a light-yellow precipitate was formed after stirring for $10 \mathrm{~min}$. The precipitate was collected and dried for $12 \mathrm{~h}$ under vacuum. For the $\left(\mathrm{FAPbI}_{3}\right)_{0.95}\left(\mathrm{MAPbBr}_{3}\right)_{0.05}$. DMSO Lewis base-additive adduct, the same procedure was repeated while a corresponding amount of FDA or CDA $(0.2 \mathrm{mmol})$ was added to the solution.

\section{Device characterization:}

The current-voltage characteristics of the solar cells were measured using a solar simulator (Newport Oriel Solar 3A Class AAA, 64023A) and a potentiostat (CHI 660D, CH instruments); the measurements were conducted under illumination of AM 1.5 G spectrum (100 $\mathrm{mA} \mathrm{cm}-2)$, and the potentiostat was calibrated using a standard Si-solar cell (Oriel, VLSI standards) and a light sensor current controller (Newport Oriel digital exposure controller, Model 68945). All devices were measured by masking the active area with a thin mask $\left(0.14 \mathrm{~cm}^{2}\right) . J-V$ curves were measured in reverse (RS) or forward scan (FS) with a scan rate of $200 \mathrm{mV} \mathrm{s}^{-1}$ under simulated AM $1.5 \mathrm{G}$ one sun illumination $\left(100 \mathrm{~mW} \mathrm{~cm}^{-2}\right)$ in ambient air (room temperature and humidity controlled at 5-10\%). We typically did light-soaking the devices under one sun condition for 20-30 s before starting J-V measurement. applied the Steady-state photoluminescence (PL) spectra were measured using a fluorescence lifetime spectrometer (Quantaurus Tau C11367-12, HAMAMATSU) with excitation by a $464 \mathrm{~nm}$ laser (PLP-10, HAMAMATSU) pulsed at a frequency of $10 \mathrm{MHz}$. TRPL was detected using the time-correlated single-photon counting technique to measure spontaneous PL decay. Scanning electron microscopy (SEM) images and elemental contents were obtained using a JSM-7600F hot field emission scanning electron microscope (JEOL, Japan). The X-ray diffraction patterns of the perovskite films were measured using a D8 Discover instrument with $\mathrm{Cu} \mathrm{K \alpha}$ radiation $(\lambda=1.5418 \AA$ ). UV-visible spectra were measured using a UV-vis spectrometer (Lambda 45, Perkin Elmer), and the UV-Vis spectrum of the perovskite film on the FTO substrate was measured. X-ray photoelectron spectra (XPS) were collected using an ESCALAB 250 XPS system (Thermo Fisher Scientific) with Al Ka X-ray radiation (1486.6 $\mathrm{eV}$ ). The IPCE spectral measurements were obtained using an IPCE system (photovoltaic measurements) in DC mode, where the monochromatic beam was supplied with a $75 \mathrm{~W} \mathrm{Xe}$ lamp (USHIO). 

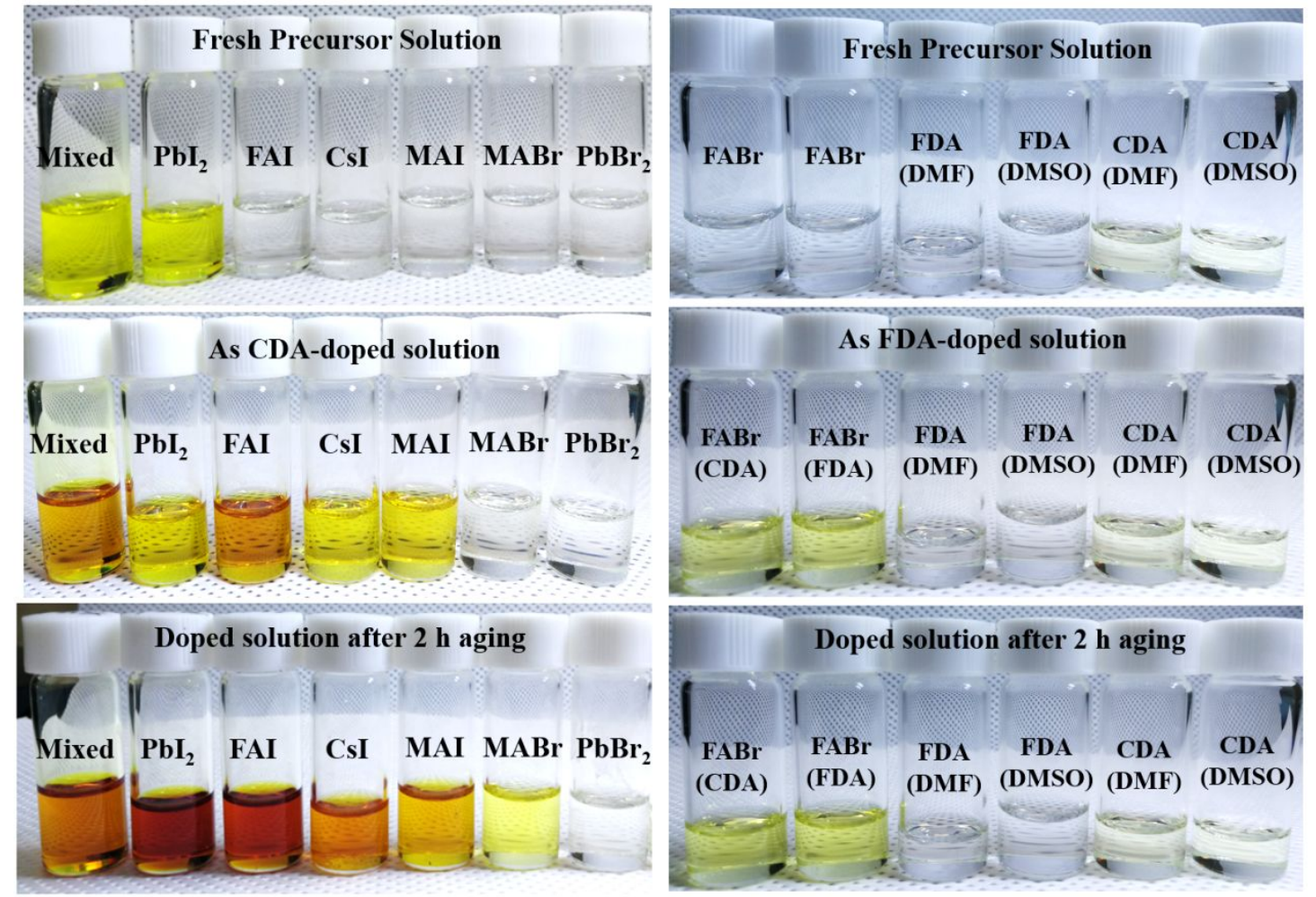

Figure S1. Images of different perovskite precursor solutions doped with CDA or FDA. 

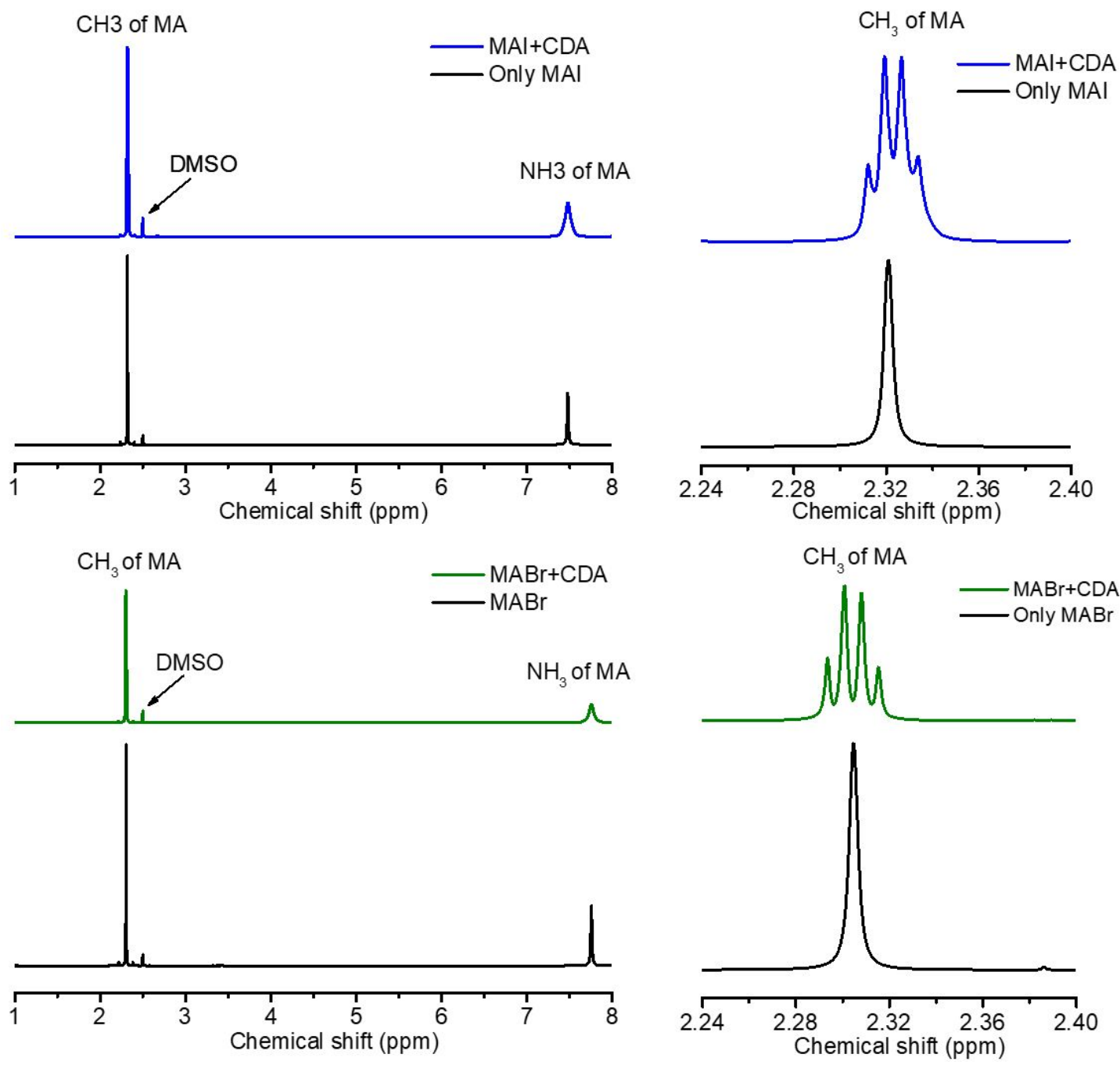

Figure S2. Corresponding liquid-state ${ }^{1} \mathrm{H}$ NMR spectra of methylammonium iodide $\left(\mathrm{CH}_{3} \mathrm{NH}_{3} \mathrm{I}(\mathrm{MAI})\right)$ or methylammonium bromide $\left(\mathrm{CH}_{3} \mathrm{NH}_{3} \mathrm{Br}\right.$ (MABr)) doped with $\mathrm{CDA}$ in deuterated DMSO- $\mathrm{d}_{6}$ solution at $295 \mathrm{~K}$. There is no peak shift or disappearance of $\mathrm{NH}_{3}$; peak splitting of $\mathrm{CH} 3$ is due to the magnetic nonequivalent ( $n+1$ rule). 


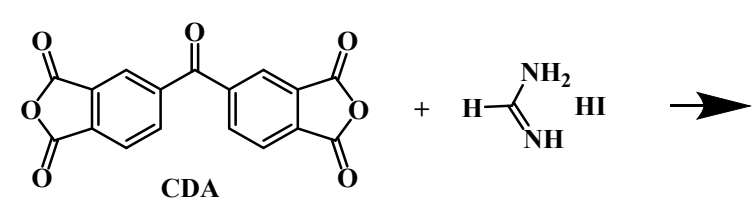<smiles>N=CNC(=O)c1ccc(C(=O)c2ccc(C(=O)NC=N)c(C(=O)O)c2)cc1C(=O)O</smiles>

a) Nucleophilic Attack by the Amine

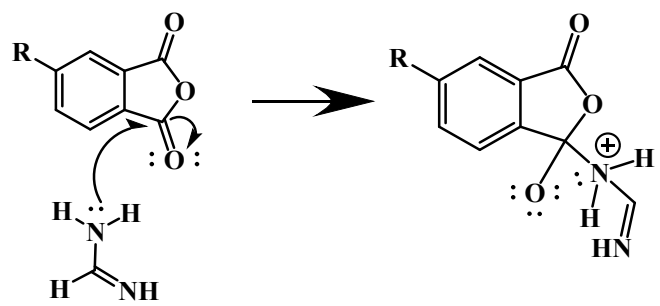

b) Deprotonation by the amine
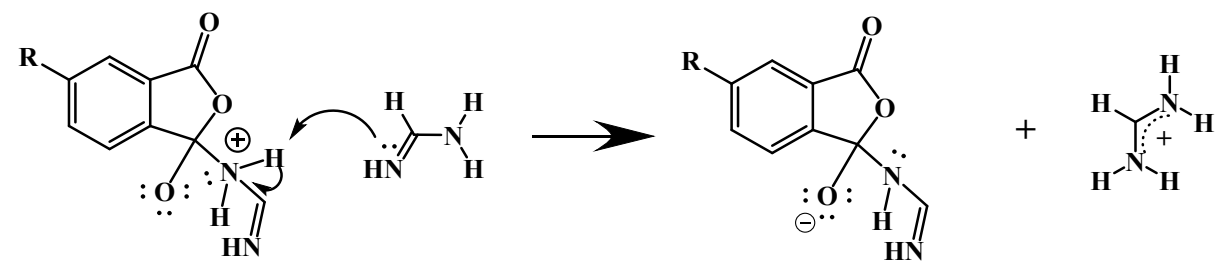

c) Leaving group removal
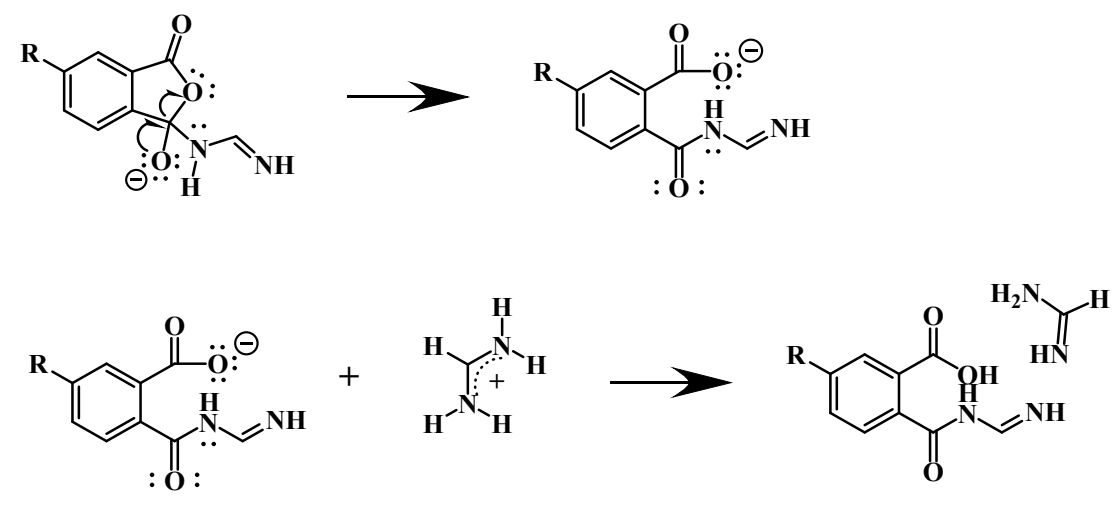

Figure S3. Representation of possible chemical interaction between FAI with CDA. 


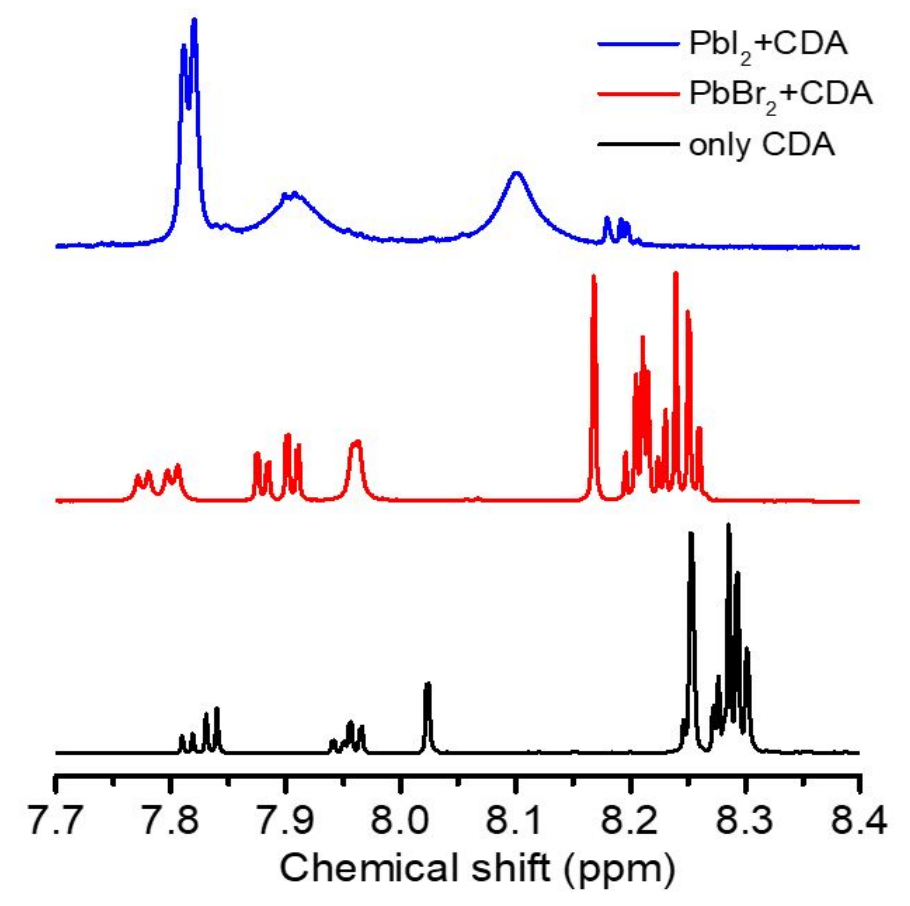

Figure S4. Corresponding liquid-state ${ }^{1} \mathrm{H}$ NMR spectra showing changes in the resonance signals of $\mathrm{PbI}_{2}$ or $\mathrm{PbBr}_{2}$ doped with CDA in deuterated DMSO-d $\mathrm{d}_{6}$ solution at $295 \mathrm{~K}$. 


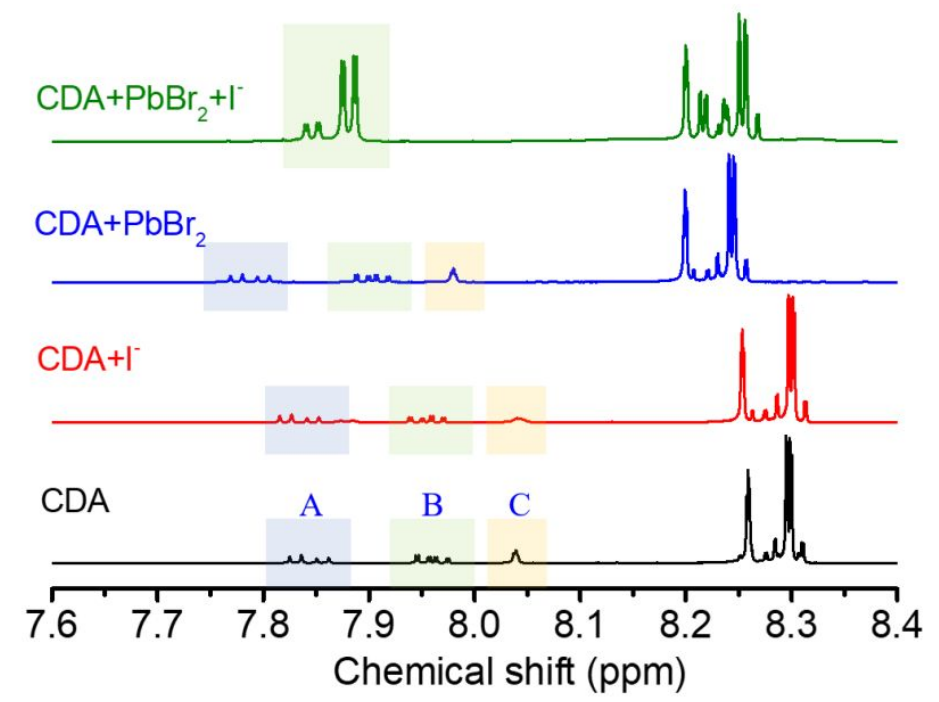

Figure S5. Corresponding liquid-state ${ }^{1} \mathrm{H}$ NMR spectra showing changes in the resonance signals of $\mathrm{CDA}, \mathrm{CDA}+\mathrm{I}^{-}, \mathrm{CDA}+\mathrm{PbBr}_{2}$ and $\mathrm{CDA}+\mathrm{PbBr}_{2}+\mathrm{I}^{-}$in deuterated $\mathrm{DMSO}-\mathrm{d}_{6}$ solution at $295 \mathrm{~K}$. 


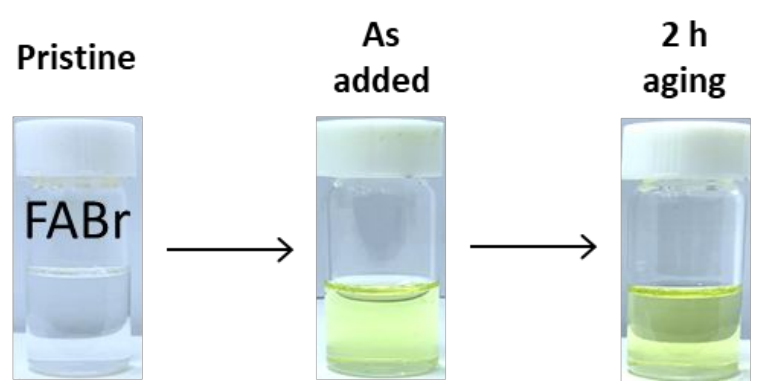

Figure S6. Images of FABr-dissolved solution with CDA doping for various aging times (as added, and $2 \mathrm{~h}$ of aging). 

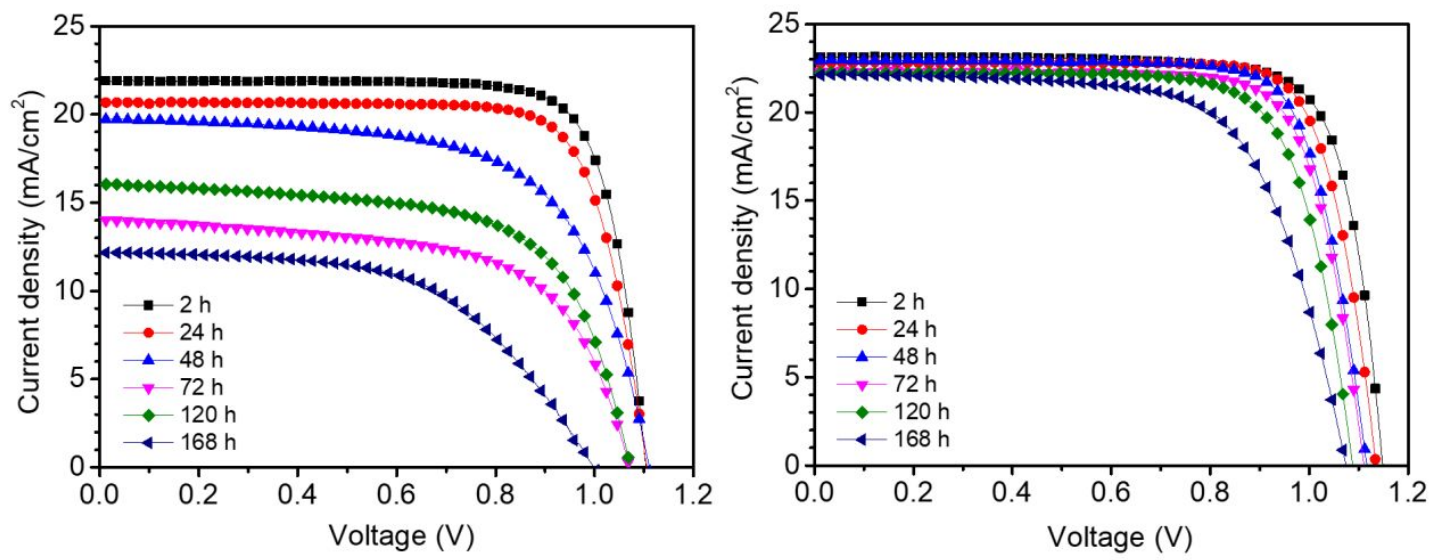

Figure S7. $J-V$ curves of PSCs (a) without and (b) with CDA as functions of the precursor aging time. 

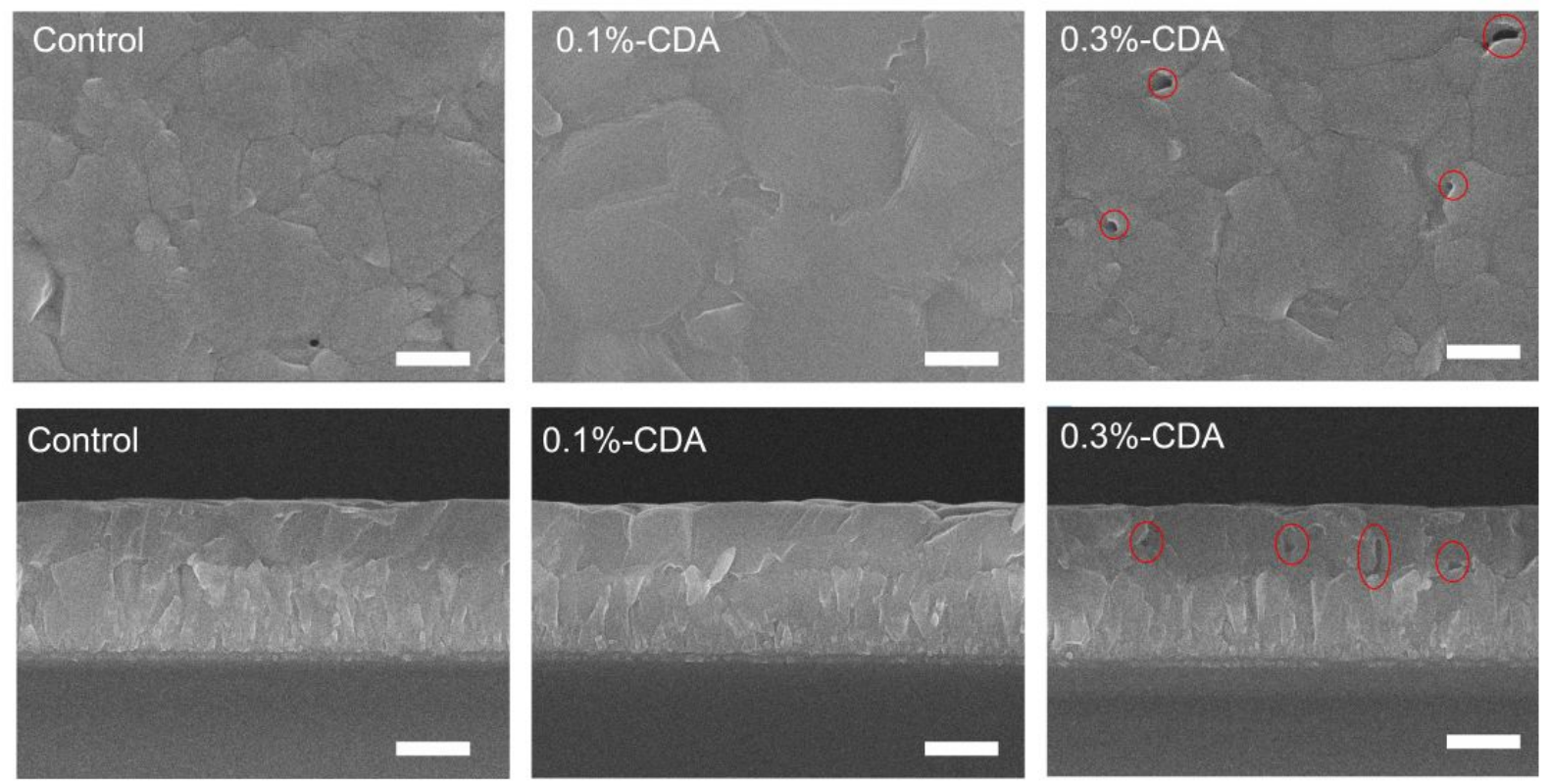

Figure S8. Top-view (top row) and cross-sectional (bottom row) SEM images of control, $0.1 \%$ CDA and $0.3 \%$ CDA films deposited onto FTO glass substrates. The scale bar in all images is $500 \mathrm{~nm}$. 

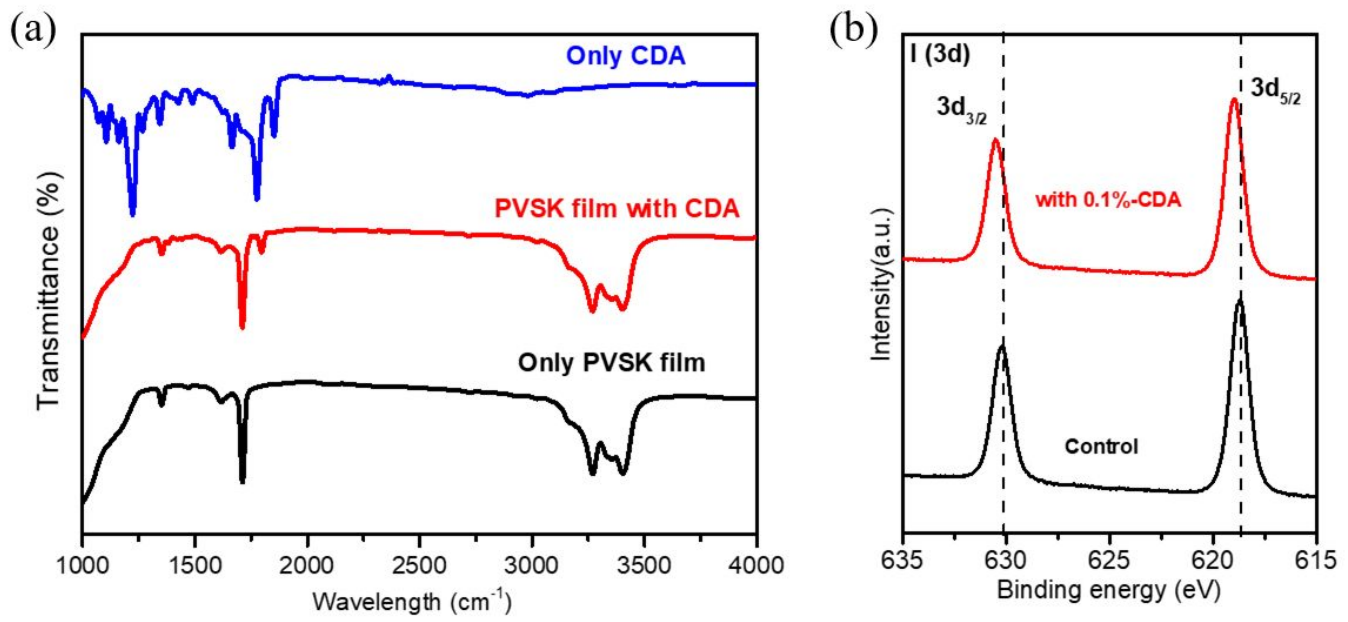

Figure S9. (a) FT-IR spectra for perovskite (PVSK) layers with or without CDA. (b) XPS spectra corresponding to I3d. (the perovskite film for the XPS spectra was deposited onto FTO glass).
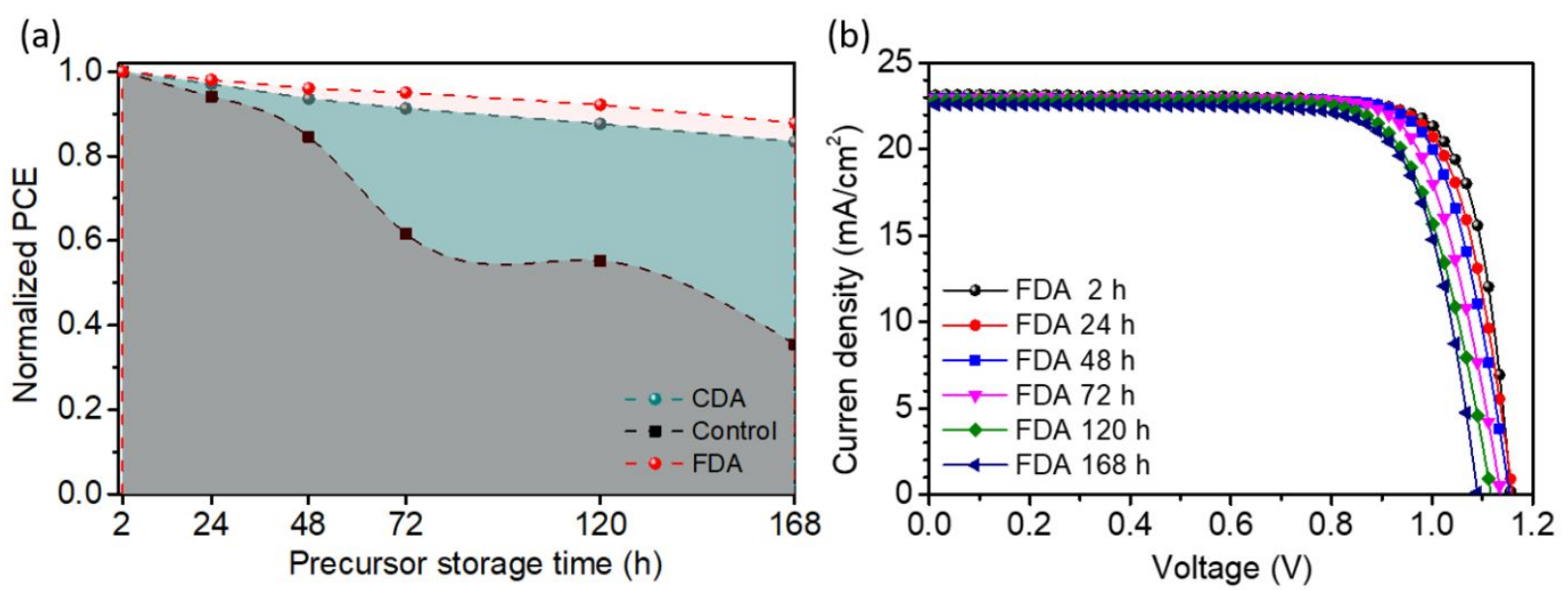

Figure S10. (a) Normalization of power conversion efficiency (PCE) for perovskite solar cells (PSCs) fabricated as a function of the storage time of precursor for control, 0.1-CDA and 0.1-FDA devices. Precursors were stored in the ambient air condition. (b) $J-V$ curves of PSCs with FDA as functions of the precursor aging time. 

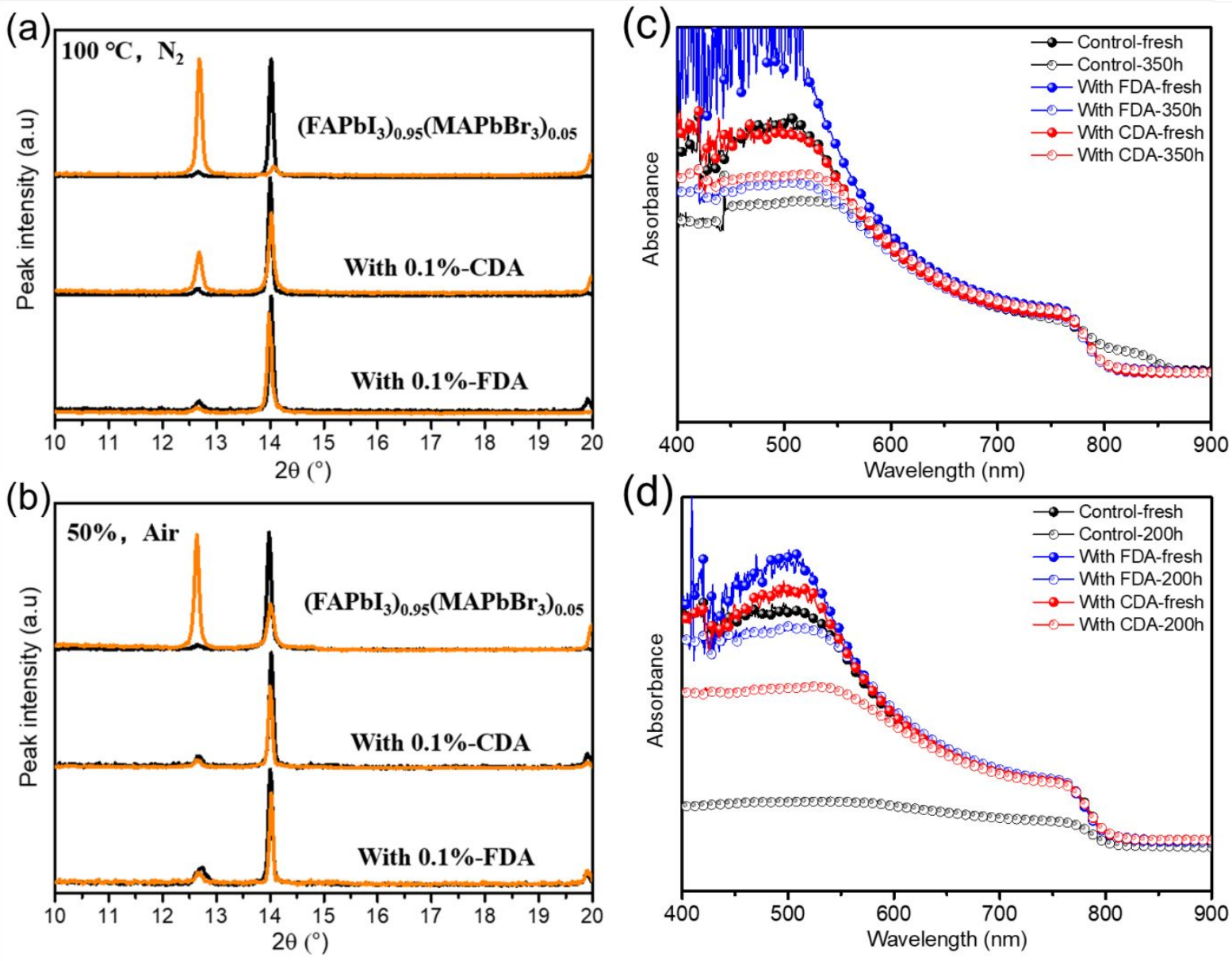

Figure S11. Stability test of the cross-linked perovskite films against (a) thermal and (b) moisture. XRD patterns of control, $0.1 \%$ CDA, and 0.1\% FDA films for pristine (black) and after aging (yellow) treatment. (c,d) Corresponding absorption spectra change for the stability test aging time. 
Thermal stability, $100^{\circ} \mathrm{C}$, in $\mathrm{N}_{2}$ atmosphere

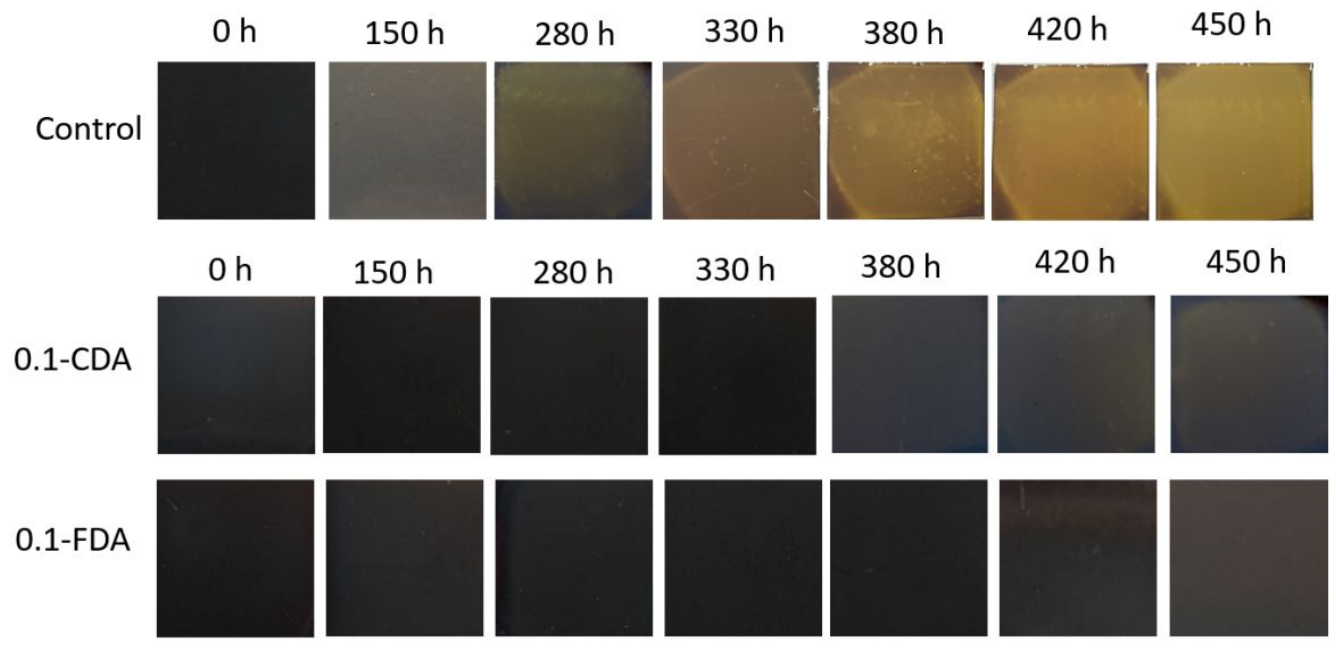

Figure S12. Thermal stability photographs of control, 0.1-CDA, and 0.1-FDA perovskite film, which were heated to $100{ }^{\circ} \mathrm{C}$ in the dark in an $\mathrm{N}_{2}$ glovebox with time 
Moisture stability (RT, RH50\%, Ambient air)

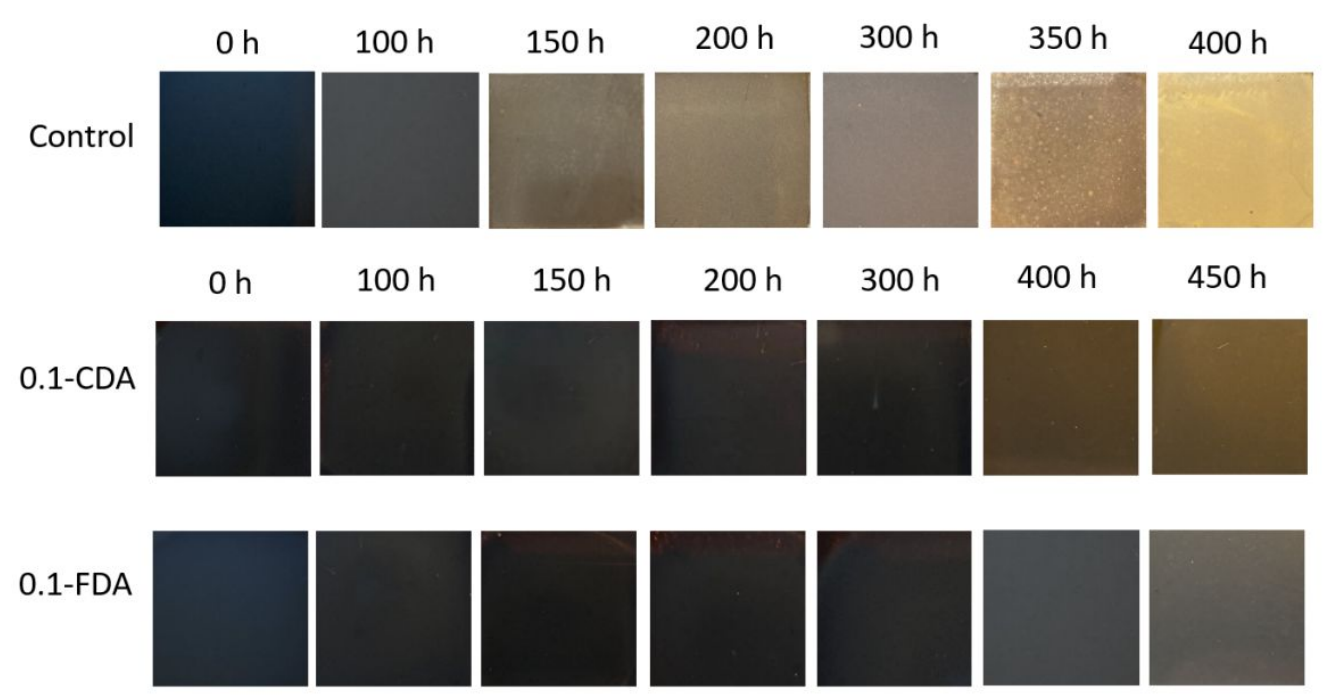

Figure S13. Moisture stability photographs of control, 0.1-CDA, and 0.1-FDA perovskite film, which the perovskite films exposed to RH $50 \pm 5 \%$ with time 


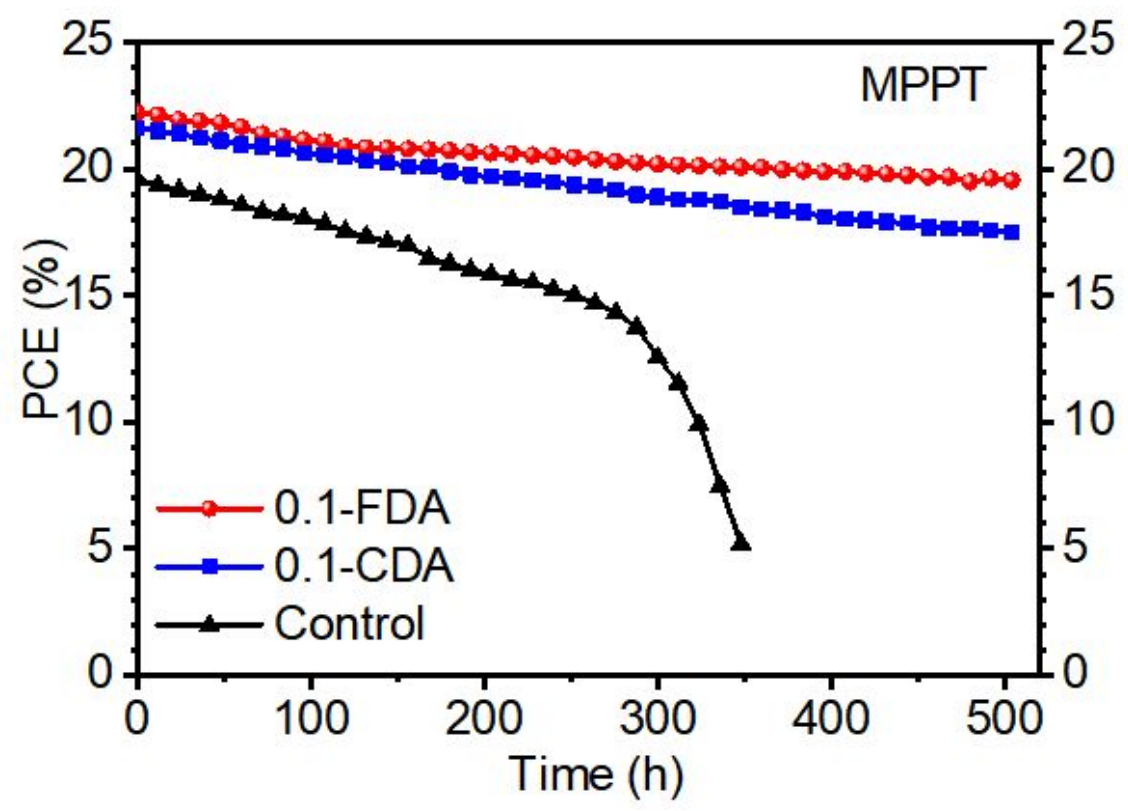

Figure S14. Maximum power point tracking under continuous illumination with LED light with intensity of $100 \mathrm{~mW} / \mathrm{cm}^{2}$ at room temperature for control, 0.1-CDA and 0.1-FDA devices without encapsulation layer. The devices were tested in $\mathrm{N}_{2}$ atmosphere at temperature ranging between 25 and $35^{\circ} \mathrm{C}$. The $J-V$ curves and the steady-state PCE at maximum power point tracking were measured every $12 \mathrm{~h}$ or $24 \mathrm{~h}$. 
Light stability, in $\mathrm{N}_{2}$ atmosphere
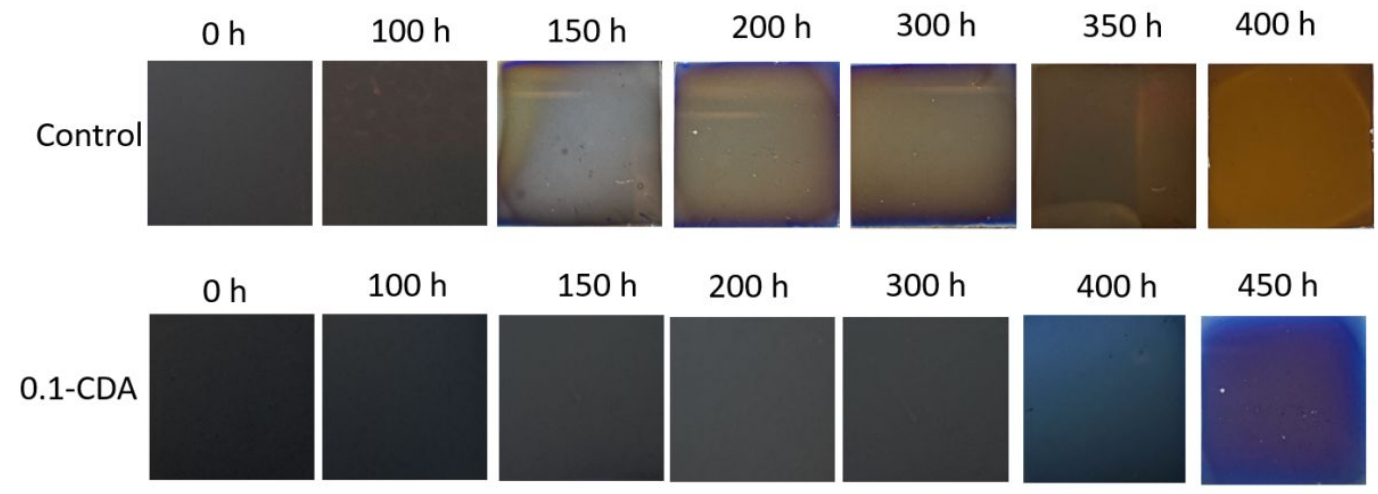

$450 \mathrm{~h}$
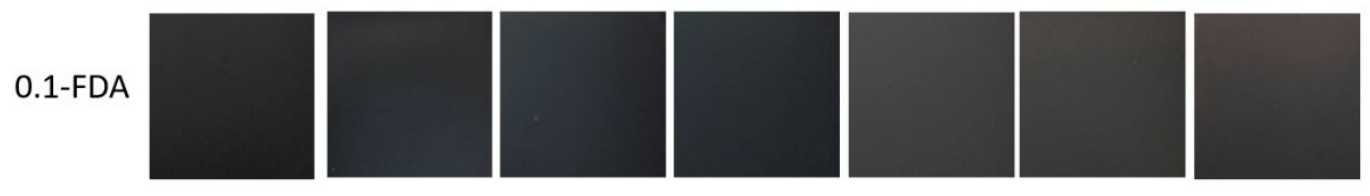

Figure S15. Light stability photographs of control, 0.1-CDA, and 0.1-FDA perovskite film under continuous 1 sun illumination (white LED, $100 \mathrm{~mW} \mathrm{~cm}^{-2}$ ) in $\mathrm{N}_{2}$ atmosphere. 

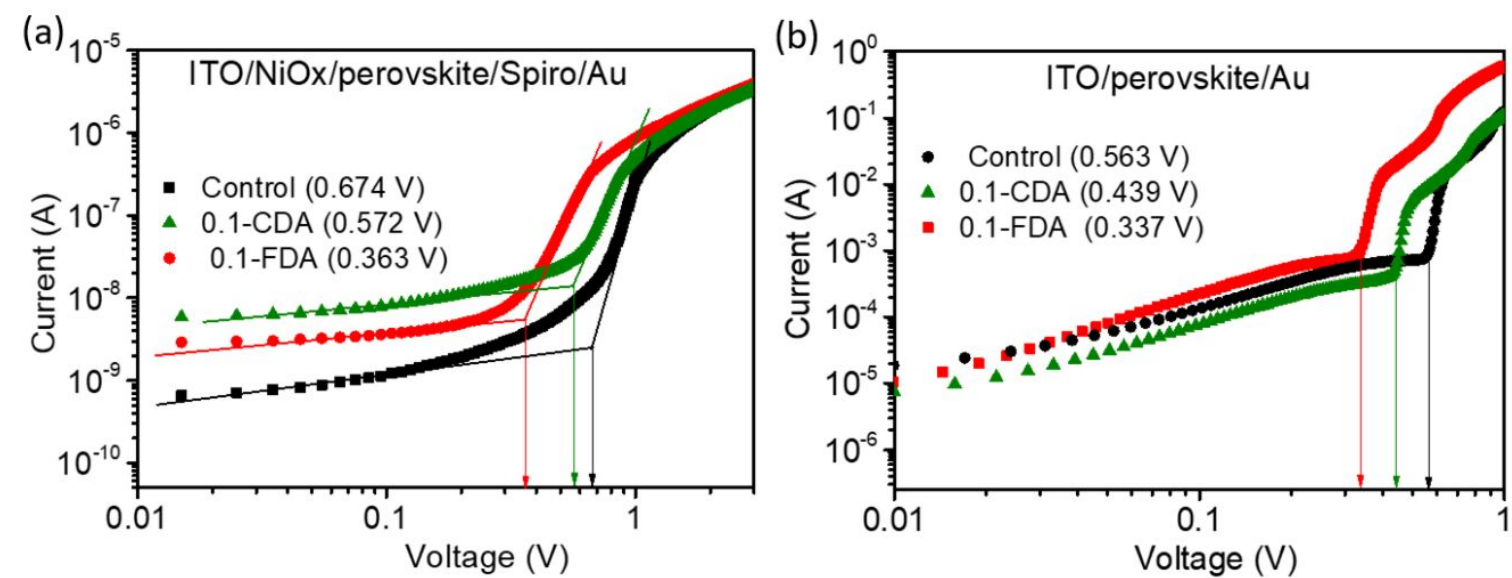

Figure S16. Space-charge-limited current (SCLC) characteristics of the control and 0.1-CDA and FDA doped perovskite with (a) ITO/NiOx/perovskite/Spiro/Au and (b) ITO/perovskite/Au device structure. 


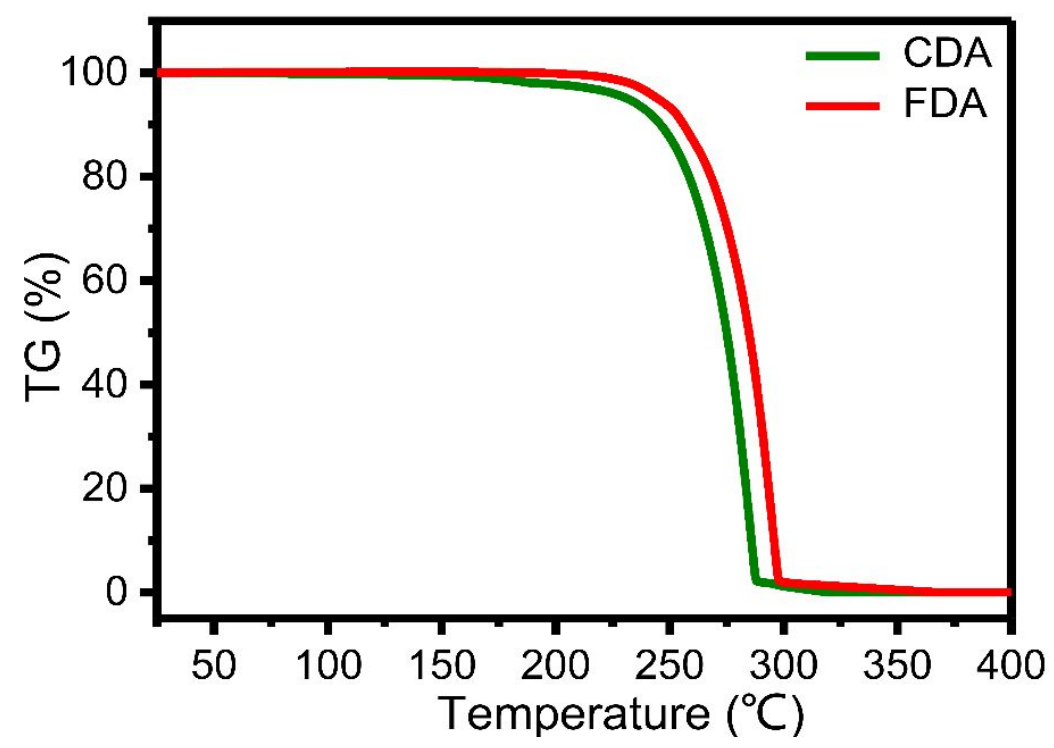

Figure S17. Thermogravimetric analysis of pure FDA and CDA. 
Table S1. Photovoltaic performance parameters of devices containing different mole ratios of CDA additives. The statistical data were collected from 15 cells for each concentration. $J-V$ curves were measured in reverse scan (RS) with the scan rate of $100 \mathrm{mV} \mathrm{s}^{-1}$ under simulated AM $1.5 \mathrm{G}$ illumination spectrum of $100 \mathrm{~W} \mathrm{~cm}^{-2}$. PCE: power conversion efficiency, FF: fill factor.

\begin{tabular}{|c|c|c|c|c|c|}
\hline \multicolumn{2}{|c|}{ Mole ratio of CDA } & $\begin{array}{c}J_{\mathrm{SC}} \\
\left(\mathrm{mA} \mathrm{cm}^{-2}\right)\end{array}$ & $\begin{array}{c}V_{\mathrm{OC}} \\
(\mathrm{V})\end{array}$ & $\mathrm{FF}$ & $\begin{array}{c}\text { PCE } \\
(\%)\end{array}$ \\
\hline \multirow{2}{*}{$0 \%$} & Champion & 22.52 & 1.12 & 0.78 & 19.58 \\
\cline { 2 - 6 } & Average & $22.36 \pm 0.211$ & $1.11 \pm 0.01$ & $0.76 \pm 0.01$ & $18.96 \pm 0.39$ \\
\hline \multirow{2}{*}{$0.05 \%$} & Champion & 22.97 & 1.14 & 0.79 & 20.82 \\
\cline { 2 - 7 } & Average & $22.87 \pm 0.19$ & $1.13 \pm 0.01$ & $0.79 \pm 0.01$ & $20.45 \pm 0.38$ \\
\hline \multirow{2}{*}{$0.1 \%$} & Champion & 23.56 & 1.15 & 0.81 & 21.88 \\
\cline { 2 - 7 } & Average & $23.03 \pm 0.35$ & $1.15 \pm 0.01$ & $0.80 \pm 0.01$ & $21.12 \pm 0.38$ \\
\hline \multirow{2}{*}{$0.3 \%$} & Champion & 22.49 & 1.13 & 0.77 & 19.50 \\
\cline { 2 - 7 } & Average & $22.78 \pm 0.16$ & $1.12 \pm 0.01$ & $0.75 \pm 0.01$ & $19.00 \pm 0.32$ \\
\hline
\end{tabular}


Table S2. Photovoltaic performance parameters of devices containing different mole ratios of FDA additives. The statistical data were collected from 15 cells for each concentration. $J-V$ curves were measured in RS with the scan rate of $100 \mathrm{mV} \mathrm{s}^{-1}$ under simulated AM $1.5 \mathrm{G}$ illumination spectrum of $100 \mathrm{~W} \mathrm{~cm}^{-2}$. HI: hysteresis index.

\begin{tabular}{|c|c|c|c|c|c|c|}
\hline \multirow{2}{*}{ Device } & $\begin{array}{c}\text { Scan } \\
\text { direction }\end{array}$ & $\begin{array}{c}J_{\mathrm{sc}} \\
\left(\mathrm{mA} \mathrm{cm}^{-2}\right)\end{array}$ & $\begin{array}{c}\text { Voc } \\
(\mathrm{V})\end{array}$ & FF & $\begin{array}{c}\text { PCE } \\
(\%)\end{array}$ & HI \\
\cline { 1 - 6 } $\begin{array}{c}0.1 \% \text {-FDA } \\
\text { (Mole ratio) }\end{array}$ & FS & 23.78 & 1.15 & 0.79 & 21.68 & \multirow{2}{*}{0.03} \\
\cline { 2 - 6 } $\begin{array}{c}0.1 \% \text {-CDA } \\
\text { (Mole ratio) }\end{array}$ & FS & 23.78 & 1.16 & 0.82 & 22.43 & \multirow{2}{*}{0.04} \\
\cline { 2 - 6 } & $\mathrm{RS}$ & 23.56 & 1.15 & 0.81 & 21.88 & \\
\hline
\end{tabular}


Table S3. Fitted parameters for the time-resolved photoluminescence decay profile of each perovskite film.

\begin{tabular}{|c|c|c|c|c|c|}
\hline Type & $\begin{array}{c}A_{1} \\
(\%)\end{array}$ & $\begin{array}{c}\tau_{1} \\
(\mathrm{~ns})\end{array}$ & $\begin{array}{c}A_{2} \\
(\%)\end{array}$ & $\begin{array}{c}\tau_{2} \\
(\mathrm{~ns})\end{array}$ & $\begin{array}{c}\tau_{\text {average }} \\
(\mathrm{ns})\end{array}$ \\
\hline Control & 70.2 & 13.8 & 29.7 & 63.9 & 46.9 \\
\hline 0.1 -FDA & 37.5 & 24.3 & 62.5 & 233.8 & 221.5 \\
\hline $0.1-\mathrm{CDA}$ & 37.1 & 27.4 & 62.9 & 189.8 & 177.1 \\
\hline
\end{tabular}


Table S4. Perovskite solar cell stability and photovoltaic performance with different Lewis additive.

\begin{tabular}{|c|c|c|c|c|c|c|}
\hline & Passivator & Device configuration & $\begin{array}{l}\mathrm{PCE} \\
(\%)\end{array}$ & $\begin{array}{l}\text { Stability } \\
(\tau 90)\end{array}$ & $\begin{array}{l}\text { measure } \\
\text { condition }\end{array}$ & Ref. \\
\hline 1 & KI & 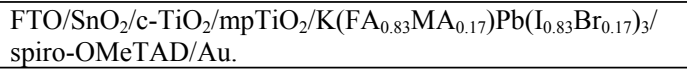 & 19.50 & $\sim 230 \mathrm{~h}$ & $\begin{array}{c}25^{\circ} \mathrm{C} \\
\mathrm{RH} 40 \%\end{array}$ & {$[1]$} \\
\hline 2 & $\mathrm{NaF}$ & $\begin{array}{l}\mathrm{ITO} / \mathrm{SnO}_{2} /\left(\mathrm{Cs}_{0.05} \mathrm{FA}_{0.54} \mathrm{MA}_{0.41}\right) \mathrm{Pb}\left(\mathrm{I}_{0.98} \mathrm{Br}_{0.02}\right)_{3} / \text { Spiro- } \\
\text { OMeTAD } / \mathrm{Au}\end{array}$ & 21.92 & $\sim 850 \mathrm{~h}$ & $85^{\circ} \mathrm{C}, \mathrm{N}_{2}$ & {$[2]$} \\
\hline 3 & TAA & $\mathrm{ITO} / \mathrm{SnO}_{2} / \mathrm{MAPbI}_{3} / \mathrm{Spiro-OMeTAD} / \mathrm{Au}$ & 15.87 & $\sim 500 \mathrm{~h}$ & $\begin{array}{c}20^{\circ} \mathrm{C} \\
\mathrm{RH} 20 \% \\
\end{array}$ & [3] \\
\hline 4 & $\begin{array}{c}1,3- \\
\text { diaminopropane } \\
\end{array}$ & ITO/PTAA/MAPbI $/ 3 / \mathrm{C} 60 / \mathrm{BCP} / \mathrm{Cu}$ & 21.70 & $>1000 \mathrm{~h}$ & $\begin{array}{c}\text { RT, } \\
\mathrm{RH} \sim 50 \% \\
\end{array}$ & {$[4]$} \\
\hline 5 & ITIC & $\mathrm{FTO} / \mathrm{c}-\mathrm{TiO}_{2} / \mathrm{MAPbI}_{3} /$ Spiro-OMeTAD/Au & 19.04 & $\sim 750$ & $\begin{array}{c}\text { RT, } \\
\text { RH } \sim 50 \%\end{array}$ & {$[5]$} \\
\hline 6 & Theophylline & $\mathrm{ITO} / \mathrm{SnO}_{2} /\left(\mathrm{FAPbI}_{3}\right) \times\left(\mathrm{MAPbBr}_{3}\right) 1 \times /$ Spiro-OMeTAD $/ \mathrm{Ag}$ & 23.48 & $>500 \mathrm{~h}$ & $\begin{array}{c}40^{\circ} \mathrm{C} \\
\mathrm{RH} 40 \%\end{array}$ & {$[6]$} \\
\hline 7 & AQ310 & $\begin{array}{l}\mathrm{FTO} / \mathrm{c}-\mathrm{TiO}_{2} / \mathrm{m}-\mathrm{TiO}_{2} /\left(\mathrm{FAPbI}_{3}\right)_{0.85}\left(\mathrm{MAPbBr}_{3}\right)_{0.15} / \text { Spiro- } \\
\text { OMeTAD } / \mathrm{Ag}\end{array}$ & 19.66 & $\sim 300 \mathrm{~h}$ & $\begin{array}{c}\text { RT, } \\
\text { RH } \sim 30 \%\end{array}$ & [7] \\
\hline 8 & PAGG & $\mathrm{ITO} / \mathrm{SnO}_{2} / \mathrm{FA}_{1-\mathrm{x}} \mathrm{MA}_{\mathrm{x}} \mathrm{PbI}_{3} /$ Spiro-OMeTAD/Ag & 23 & $\sim 500 \mathrm{~h}$ & $\begin{array}{l}\text { lightsoaking } \\
\text { RH } \sim 35 \% \\
\end{array}$ & {$[8]$} \\
\hline 9 & ТВРO & $\begin{array}{l}\mathrm{FTO} / \mathrm{c}-\mathrm{TiO}_{2} / \mathrm{PCBA} /\left(\mathrm{FAPbI}_{3}\right)_{0.87}\left(\mathrm{MAPbBr}_{3}\right)_{0.13} / \text { Spiro- } \\
\mathrm{OMeTAD} / \mathrm{Au}\end{array}$ & 21.70 & $\sim 25 \mathrm{~h}$ & $\begin{array}{c}60^{\circ} \mathrm{C} \\
\mathrm{RH} 60 \%\end{array}$ & [9] \\
\hline 10 & $\begin{array}{l}\text { 3-(1-Pyridinio)-1- } \\
\text { propanesulfonate }\end{array}$ & $\mathrm{FTO} / \mathrm{SnO}_{2} / \mathrm{CsMAFA} / \mathrm{Spiro}-\mathrm{OMeTAD} / \mathrm{Au}$ & 21.43 & $\sim 140 \mathrm{~h}$ & $\begin{array}{c}85^{\circ} \mathrm{C} \\
\mathrm{RH} 85 \%\end{array}$ & {$[10]$} \\
\hline 11 & MSAPBS & $\begin{array}{l}\mathrm{ITO} / \mathrm{SnO}_{2} / \mathrm{Cs}_{0.05} \mathrm{FA}_{0.81} \mathrm{MA}_{0.14} \mathrm{PbI}_{2.55} \mathrm{Br}_{0.45} / \text { Spiro-OMeTAD } \\
/ \mathrm{Au}\end{array}$ & 21.18 & $\sim 500 \mathrm{~h}$ & $\begin{array}{c}25^{\circ} \mathrm{C} \\
\mathrm{RH} 25 \%\end{array}$ & [11] \\
\hline 12 & DPSI & ITO/PTAA/FA ${ }_{0.85} \mathrm{MA}_{0.15} \mathrm{~Pb}\left(\mathrm{Br}_{0.15} \mathrm{I}_{0.85}\right)_{3} / \mathrm{PCBM} / \mathrm{C}_{60} / \mathrm{BCP} / \mathrm{Cu}$ & 21.10 & $\sim 480 \mathrm{~h}$ & $\begin{array}{c}\text { under one } \\
\text { sun }\end{array}$ & [12] \\
\hline 13 & $\mathrm{PPC}$ & $\mathrm{ITO} / \mathrm{SnO}_{2} / \mathrm{MAPbI}_{3} /$ Spiro-MeOTAD/Ag. & 20.06 & $>1000 \mathrm{~h}$ & $\begin{array}{c}\text { ambient } \\
\text { conditions }\end{array}$ & [13] \\
\hline 14 & Caffeine & $\mathrm{ITO} / \mathrm{SnO}_{2} / \mathrm{MAPbI}_{3} /$ Spiro-MeOTAD/Ag. & 20.25 & $\sim 1000 \mathrm{~h}$ & $85^{\circ} \mathrm{C}, \mathrm{N}_{2}$ & [14] \\
\hline 15 & $2,2^{\prime}$-bipyridine & $\mathrm{FTO} / \mathrm{TiO}_{2} / \mathrm{FA}_{0.88} \mathrm{Cs}_{0.12} \mathrm{PbI}_{3} /$ spiro-OMeTAD/Au. & 20.56 & $\sim 220 \mathrm{~h}$ & RH $50 \sim 70 \%$ & {$[15]$} \\
\hline 16 & $\begin{array}{l}\text { (3-mercaptopropy } \\
\text { 1)trimethoxysilane }\end{array}$ & FTO/SnO $2 /$ perovskite/Spiro-OMeTAD/Ag & 20.81 & $\sim 350 \mathrm{~h}$ & $85^{\circ} \mathrm{C}, \mathrm{N}_{2}$ & [16] \\
\hline 17 & $\begin{array}{c}\text { polymer poly } \\
(4-v i n y l p y r i d i n e)\end{array}$ & $\mathrm{ITO} / \mathrm{SnO}_{2} /$ perovskite/Spiro-OMeTAD/Au & 20.23 & $\sim 1000 \mathrm{~h}$ & $\begin{array}{l}20^{\circ} \sim 25^{\circ} \mathrm{C} \\
\mathrm{RH} 25 \sim 40 \%\end{array}$ & [17] \\
\hline 18 & Caprolactam & $\mathrm{FTO} / \mathrm{TiO}_{2} /$ perovskite/Spiro-OMeTAD/Ag & 19.2 & $\sim 1200 \mathrm{~h}$ & $\begin{array}{l}\text { ambient } \\
\text { condition }\end{array}$ & [18] \\
\hline 19 & $\mathrm{NbF}_{5}$ & $\mathrm{FTO} / \mathrm{TiO}_{2} / \mathrm{FA}_{0.85} \mathrm{~A}_{0.15} \mathrm{PbI}_{3} /$ spiro-OMeTAD/Au. & 20.56 & $\sim 480 \mathrm{~h}$ & $\mathrm{RH} \sim 40 \%$ & [19] \\
\hline \multirow{2}{*}{$\begin{array}{l}\text { This } \\
\text { work }\end{array}$} & FDA & $\mathrm{FTO} / \mathrm{TiO}_{2} /\left(\mathrm{FAPbI}_{3}\right)_{0.95}\left(\mathrm{MAPbBr}_{3}\right)_{0.05} /$ Spiro-OMeTAD/Au & \multirow{2}{*}{22.43} & $\sim 900 \mathrm{~h}$ & \multicolumn{2}{|c|}{$80^{\circ} \mathrm{C}, \mathrm{N}_{2}$} \\
\hline & FDA & $\mathrm{FTO} / \mathrm{TiO}_{2} /\left(\mathrm{FAPbI}_{3}\right)_{0.95}\left(\mathrm{MAPbBr}_{3}\right)_{0.05} /$ Spiro-OMeTAD $/ \mathrm{Au}$ & & $\sim 800 \mathrm{~h}$ & \multicolumn{2}{|c|}{$\begin{array}{c}\text { RT, } \\
\text { RH } 50 \%\end{array}$} \\
\hline
\end{tabular}




\section{Reference}

(1) Yang, Y.;Wu, L.;Hao, X.;Tang, Z.;Lai, H.;Zhang, J.;Wang, W.;Feng, L., Beneficial effects of potassium iodide incorporation on grain boundaries and interfaces of perovskite solar cells. RSC Advances 2019, 9 (49), 28561.

(2) Li, N.;Tao, S.;Chen, Y.;Niu, X.;Onwudinanti, C. K.;Hu, C.;Qiu, Z.;Xu, Z.;Zheng, G.;Wang, L.;Zhang, Y.;Li, L.;Liu, H.;Lun, Y.;Hong, J.;Wang, X.;Liu, Y.;Xie, H.;Gao, Y.;Bai, Y.;Yang, S.;Brocks, G.;Chen, Q.;Zhou, H., Cation and anion immobilization through chemical bonding enhancement with fluorides for stable halide perovskite solar cells. Nature Energy 2019, 4 (5), 408.

(3) Jeong, J.;Kim, M.;Seo, J.;Lu, H.;Ahlawat, P.;Mishra, A.;Yang, Y.;Hope, M. A.;Eickemeyer, F. T.;Kim, M.;Yoon, Y. J.;Choi, I. W.;Darwich, B. P.;Choi, S. J.;Jo, Y.;Lee, J. H.;Walker, B.;Zakeeruddin, S. M.;Emsley, L.;Rothlisberger, U.;Hagfeldt, A.;Kim, D. S.;Grätzel, M.;Kim, J. Y., Pseudo-halide anion engineering for $\alpha$-FAPbI3 perovskite solar cells. Nature 2021, 592 (7854), 381.

(4) Wu, W.-Q.;Yang, Z.;Rudd, P. N.;Shao, Y.;Dai, X.;Wei, H.;Zhao, J.;Fang, Y.;Wang, Q.;Liu, Y.;Deng, Y.;Xiao, X.;Feng, Y.;Huang, J., Bilateral alkylamine for suppressing charge recombination and improving stability in blade-coated perovskite solar cells. Science Advances 2019, 5 (3), eaav8925.

(5) Niu, T.;Lu, J.;Munir, R.;Li, J.;Barrit, D.;Zhang, X.;Hu, H.;Yang, Z.;Amassian, A.;Zhao, K.;Liu, S., Stable High-Performance Perovskite Solar Cells via Grain Boundary Passivation. Advanced Materials 2018, 30 (16), 1706576.

(6) Wang, R.;Xue, J.;Wang, K.-L.;Wang, Z.-K.;Luo, Y.;Fenning, D.;Xu, G.;Nuryyeva, S.;Huang, T.;Zhao, Y.;Yang, J. L.;Zhu, J.;Wang, M.;Tan, S.;Yavuz, I.;Houk, K. N.;Yang, Y., Constructive molecular configurations for surface-defect passivation of perovskite photovoltaics. Science 2019, 366 (6472), 1509.

(7) Li, X.;Chen, C.-C.;Cai, M.;Hua, X.;Xie, F.;Liu, X.;Hua, J.;Long, Y.-T.;Tian, H.;Han, L., Efficient Passivation of Hybrid Perovskite Solar Cells Using Organic Dyes with $\square \mathrm{COOH}$ Functional Group. Advanced Energy Materials 2018, 8 (20), 1800715.

(8) Zhao, Y.;Zhu, P.;Wang, M.;Huang, S.;Zhao, Z.;Tan, S.;Han, T.-H.;Lee, J.-W.;Huang, T.;Wang, R.;Xue, J.;Meng, D.;Huang, Y.;Marian, J.;Zhu, J.;Yang, Y., A PolymerizationAssisted Grain Growth Strategy for Efficient and Stable Perovskite Solar Cells. Advanced Materials 2020, 32 (17), 1907769.

(9) Li, H.;Shi, J.;Deng, J.;Chen, Z.;Li, Y.;Zhao, W.;Wu, J.;Wu, H.;Luo, Y.;Li, D.;Meng, Q., Intermolecular $\pi-\pi$ Conjugation Self-Assembly to Stabilize Surface Passivation of Highly Efficient Perovskite Solar Cells. Advanced Materials 2020, 32 (23), 1907396.

(10) Choi, K.;Lee, J.;Kim, H. I.;Park, C. W.;Kim, G.-W.;Choi, H.;Park, S.;Park, S. A.;Park, T., Thermally stable, planar hybrid perovskite solar cells with high efficiency. Energy \& Environmental Science 2018, 11 (11), 3238.

(11) Zheng, D.;Peng, R.;Wang, G.;Logsdon, J. L.;Wang, B.;Hu, X.;Chen, Y.;Dravid, V. P.;Wasielewski, M. R.;Yu, J.;Huang, W.;Ge, Z.;Marks, T. J.;Facchetti, A., Simultaneous Bottom-Up Interfacial and Bulk Defect Passivation in Highly Efficient Planar Perovskite Solar Cells using Nonconjugated Small-Molecule Electrolytes. Advanced Materials 2019, 31 (40), 1903239.

(12) Zheng, X.;Deng, Y.;Chen, B.;Wei, H.;Xiao, X.;Fang, Y.;Lin, Y.;Yu, Z.;Liu, Y.;Wang, Q.;Huang, J., Dual Functions of Crystallization Control and Defect Passivation Enabled by Sulfonic Zwitterions for Stable and Efficient Perovskite Solar Cells. Advanced Materials 2018, 30 (52), 1803428.

(13) Han, T.-H.;Lee, J.-W.;Choi, C.;Tan, S.;Lee, C.;Zhao, Y.;Dai, Z.;De Marco, N.;Lee, S.J.;Bae, S.-H.;Yuan, Y.;Lee, H. M.;Huang, Y.;Yang, Y., Perovskite-polymer composite cross- 
linker approach for highly-stable and efficient perovskite solar cells. Nature Communications 2019, 10 (1), 520.

(14) Wang, R.;Xue, J.;Meng, L.;Lee, J.-W.;Zhao, Z.;Sun, P.;Cai, L.;Huang, T.;Wang, Z.;Wang, Z.-K.;Duan, Y.;Yang, J. L.;Tan, S.;Yuan, Y.;Huang, Y.;Yang, Y., Caffeine Improves the Performance and Thermal Stability of Perovskite Solar Cells. Joule 2019, 3 (6), 1464.

(15) Jiang, Q.;Zhao, Y.;Zhang, X.;Yang, X.;Chen, Y.;Chu, Z.;Ye, Q.;Li, X.;Yin, Z.;You, J., Surface passivation of perovskite film for efficient solar cells. Nature Photonics 2019, 13 (7), 460.

(16) Xie, L.;Chen, J.;Vashishtha, P.;Zhao, X.;Shin, G. S.;Mhaisalkar, S. G.;Park, N.-G., Importance of Functional Groups in Cross-Linking Methoxysilane Additives for HighEfficiency and Stable Perovskite Solar Cells. ACS Energy Letters 2019, 4 (9), 2192.

(17) Zuo, L.;Guo, H.;deQuilettes, D. W.;Jariwala, S.;De Marco, N.;Dong, S.;DeBlock, R.;Ginger, D. S.;Dunn, B.;Wang, M.;Yang, Y., Polymer-modified halide perovskite films for efficient and stable planar heterojunction solar cells. Science Advances 2017, 3 (8), e1700106.

(18) Li, H.;Li, Y.;Li, Y.;Shi, J.;Zhang, H.;Xu, X.;Wu, J.;Wu, H.;Luo, Y.;Li, D.;Meng, Q., Synergistic effect of caprolactam as lewis base and interface engineering for efficient and stable planar perovskite solar cells. Nano Energy 2017, 42, 222.

(19) Yuan, S.;Qian, F.;Yang, S.;Cai, Y.;Wang, Q.;Sun, J.;Liu, Z.;Liu, S., NbF5: A Novel $\alpha$-Phase Stabilizer for FA-Based Perovskite Solar Cells with High Efficiency. Advanced Functional Materials 2019, 29 (47), 1807850. 